\title{
Más Allá de la Indexación: Circuitos de Publicación de Ciencias Sociales en Argentina y Brasil $^{*}$
}

\author{
Maximiliano Salatino \\ INCIHUSA-CONICET Mendoza/Universidad Nacional de Cuyo, Argentina. E-mail: \\ maxisalatino@gmail.com
}

\section{INTRODUCCIÓN}

$\mathrm{D}$ esde América Latina, concretamente a partir de 1960 y 1970, emergieron discusiones vinculadas a la ciencia y su relación con el desarrollo; ciencia y política; y, sobre todo, planteos vinculados a las posibilidades de generar conocimientos científicos y tecnológicos, desde la región, cada vez más autónomos ${ }^{1}$. En el caso concreto de las publicaciones periódicas, Hebe Vessuri (1987, 1988, 1989, 1993, 1995, 2001) inició una serie de discusiones vinculadas a la situacionalidad de la ciencia periférica y más concretamente, produjo valiosos aportes acerca de las revistas científicas y su edición en la región. Asimismo, la investigadora brasileña Léa Velho analizó las asimetrías y desigualdades de los sistemas de evaluación de la ciencia y sus consecuencias para América Latina $(1986,1992,1994)$. La mexicana Ana María Cetto desde su posición de editora de la Revista Mexicana de Física y luego como fundadora del proyecto Latindex alentó en diversos espacios (talleres, redes, reuniones de trabajo, publicaciones) la construcción de una mirada regional de las revistas científicas e impulsó políticamente la creación del primer directorio y catálogo de publicaciones latinoamericanas (Cetto, 1995, 1998, 1999). Las discusiones sobre las revistas científicas en la región ya poseen alrededor de 50 años. Los estudios

\footnotetext{
* El autor quiere agradecer los comentarios atentos y generosos de Fernanda Beigel, Rogerio Mugnaini y Osvaldo López Ruiz, así como también, las correcciones de los evaluadores ciegos de la primera versión del artículo.
}

DADOS - Revista de Ciências Sociais, Rio de Janeiro, vol. 61, no-1, 2018, pp. 255 a 287.

http://dx.doi.org/10.1590/001152582018152 
han transitado desde la necesidad de generar un diagnóstico de la comunicación científica y una exploración de las prácticas de gestión editorial hasta la emergencia de fuertes críticas a la consolidación de la evaluación cuantitativa.

Nuestro trabajo se sitúa en la perspectiva de los estudios críticos sobre dependencia académica (Alatas, 2003, 2014; Beigel, 2010, 2012) para analizar las formas en que el conocimiento científico social circula en dos países de la región: Argentina y Brasil. Sostenemos que el mundo de la ciencia se ha estructurado a nivel global a partir de una serie de jerarquizaciones y desigualdades constituidas históricamente. Estos procesos tomaron forma a partir de lo que autores como Guédon (2001, 2011), Gingras (2002) y Beigel (2014) han definido como sistema académico mundial (SAM). Esta estructura jerárquica y desigual del mundo científico se consolidó a partir de la creación de las grandes corporaciones editoriales que se encargaron de constituir una ingeniería de parámetros de evaluación de la ciencia a partir de la cual se inició una categorización de la "excelencia" por sobre la "calidad" de una revista (Vessuri, Guédon y Cetto, 2014). En este sentido, los criterios de evaluación adoptados por el Institute for Scientific Information (ISI) ${ }^{2}$ creado por Eugene Garfield en 1959 iniciaron un proceso de universalización que constituyeron las bases fundantes de la cienciometría. Alrededor de ISI-WoS se forjó una suerte de acumulación primitiva de prestigio científico que benefició a ciertas zonas geográficas, grupos idiomáticos y disciplinas, al tiempo que fue ampliando la distancia con áreas "periféricas" cada vez más desprovistas de reconocimiento internacional (Beigel, 2014). Desde este punto de partida, nos preguntamos ide qué manera la conformación del SAM ha incidido en las formas en que el conocimiento científico social circula en la región?, ¿cómo se han manifestado estas asimetrías y desigualdades en la edición de revistas científicas? Si analizamos las revistas de ciencias sociales y humanidades editadas en Argentina y Brasil, ¿la lógica de la indexación basta para dar cuenta de las complejidades de la circulación y visibilidad del conocimiento científico?

Concretamente la circulación internacional del conocimiento se materializa en redes, proyectos internacionales, movilidades académicas y otras formas de investigación colaborativa, pero son los circuitos de publicación los espacios privilegiados de consagración académica (Beigel y Salatino, 2015). La circulación de las revistas se inserta en circuitos de publicación que, en regiones periféricas como América Lati- 
Más Allá de la Indexación: Circuitos de Publicación de Ciencias Sociales...

na, adquieren características particulares y, por tanto, atraviesan los campos científicos nacionales a partir de diversas estrategias.

Desarrollamos el concepto de circuito de publicación como una herramienta analítica que nos permite examinar la circulación de las ciencias sociales y humanidades en el seno de las instituciones editoras, los repositorios y bases indexadoras, las políticas de gestión editorial y las particularidades disciplinares. A partir de este concepto buscamos avanzar más allá de la lógica de la indexación para indagar los procesos estructurantes alrededor del prestigio y la calidad de una revista científica. Es que:

Pensar en términos de circuitos implica deshacer la identificación típicamente extendida entre visibilidad e indexación, puesto que la circulación del conocimiento depende de su accesibilidad. Una revista puede estar indexada en sistemas mainstream que otorgan importantes recompensas en términos de legitimación para la trayectoria individual de un científico, pero tendrá una circulación básicamente limitada a los centros de excelencia (Beigel y Salatino, 2015:14).

Por tanto, avanzar hacia la definición empírica de circuitos de publicación permite una mirada más aguda en torno al alcance real e impacto del conocimiento científico.

Para este trabajo se construyó una base de datos con registros de publicaciones científicas periódicas de ciencias sociales y humanidades activas al año 2015 editadas en Argentina y Brasil ${ }^{3}$. El total de publicaciones registradas es de 4459, de las cuales 3609 corresponden a Brasil y 850 a la Argentina. Para su construcción se relevaron los datos de Latindex Directorio, base considerada como la más abarcativa de toda la región y que incluye aquellas publicaciones que poseen algún interés académico. A partir de la depuración y guiados por las series de ISSN de los dos países se realizó el cruzamiento con otras bases y repositorios tales como Latindex Catálogo, SciELO, RedALyC, DOAJ, DIALNET, Web of Science (Social Science Citation Index y Arts and Humanities Citation Index), SCOPUS y Ulrich's Periodical Directory ${ }^{4}$. Asimismo, se tuvo en consideración los listados CAPES-QUALIS y el Núcleo Básico de Revistas del CAICYT (Centro Argentino de Información Científica y Tecnológica - CONICET).

La construcción de la base fue guiada a partir de un conjunto de variables que buscaron caracterizar las disciplinas y áreas temáticas, fechas

DADOS - Revista de Ciências Sociais, Rio de Janeiro, vol. 61, nº 1, 2018 
de creación (en formato papel y digital), la institución editora, la regularidad, su disponibilidad en texto completo, el idioma, el soporte y su indexación. A partir de este relevamiento, se logró relacionar diferentes elementos que han modelado la edición y circulación de revistas en ambos países. Se analizaron los datos a través de tres coeficientes de correlación (T-de Schuprow, V de Cramer y Ji-Cuadrado) para la obtención de asociaciones que nos permitan generar síntesis estadísticas (Test-Values). Se utilizó para el procesamiento el software Coheris Analytics SPAD 8.

En el análisis general de las revistas identificamos diferenciaciones vinculadas principalmente a las instituciones editoras, la accesibilidad, las disciplinas y la indexación. Para ello, se tuvo en consideración los resultados de los coeficientes inferenciales y los análisis estadísticos descriptivos integrales de la base de revistas estudiadas. En función de estas segmentaciones, construimos analíticamente cuatro circuitos de publicaciones que representan diferentes prácticas editoriales y científicas.

El trabajo se divide en tres secciones. En la primera, se indaga el proceso mediante el cual las publicaciones periódicas se constituyeron en uno de los principales instrumentos de consagración académica en el marco del sistema académico mundial. Luego, analizamos las principales características de las revistas estudiadas con el fin de detectar patrones de agrupamiento y criterios de diferenciación. En la última sección, nos proponemos estudiar empíricamente la idea de circuitos de publicación. Buscamos caracterizar estos espacios de circulación en relación con el tipo de revistas que allí se insertan, las principales áreas disciplinarias, las instituciones editoras y las modalidades de acceso/visibilidad de sus producciones.

\section{LAS REVISTAS CIENTÍFICAS COMO AGENTES DE CONSAGRACIÓN ACADÉMICA}

En el contexto mundial de institucionalización y creciente regionalización del conocimiento científico, las revistas se transformaron en uno de los principales órganos de la comunicación científica. Paulatinamente, este rol comenzó a tensionarse a partir de la consideración de las revistas como "gatekeepers" de la ciencia (Crane, 1967). Es decir, se transformaron en instrumentos que actúan como llaves de acceso a ciertos y determinados mecanismos de visibilidad, autoridad y presti- 
Más Allá de la Indexación: Circuitos de Publicación de Ciencias Sociales...

gio científico. El hecho de que una institución edite una revista considerada en su campo como "prestigiosa" trae beneficios (materiales y simbólicos) tanto a la institución en sí como a sus agentes y más directamente a su editor/a. Este posicionamiento de las revistas en el mundo de la ciencia se complejizó al punto de valorar la práctica científica con relación a sus modalidades de publicación.

Las publicaciones periódicas formaron parte de una estrategia corporativa sustanciada en un nuevo régimen de evaluación de los/as científicos / as y sus producciones. La evaluación cuantitativa de la producción científica comenzó a instalarse fuertemente como indicador de "excelencia", por tanto, como indicador fundamental de prestigio y autoridad científica. En el siglo XXI, la expansión de las tecnologías de la comunicación y la información (TICs) profundizó estos procesos a nivel mundial modificando fuertemente las formas en que la ciencia se comunica. Los cambios en las vías de comunicación científica, la expansión de experiencias de trabajo colaborativas, la constitución de un sistema editorial oligopólico, el posicionamiento de la "cultura del paper", la expansión de la digitalización y, más concretamente, la adopción de recursos cienciométricos ${ }^{5}$ produjeron cambios estructurales que plantearon nuevas reglas en la relación entre el sistema de publicaciones y las leyes del mercado científico.

La tensión global/local se refleja en las nuevas modalidades de evaluación de las producciones científicas de individuos e instituciones. Los rankings de universidades y el desarrollo de la cienciometría son parte fundamental del estado actual de competencia en las ciencias sociales. Muchos autores destacan las limitaciones de dichos rankings y las restricciones de la cienciometría (Vessuri, Guédon y Cetto, 2014; Heilbron, 2012; Guédon, 2008; Ortiz, 2009; Vessuri, 1997) pero a pesar de ello, ambas herramientas continúan un camino de consolidación. Esto ha generado diferentes fenómenos que no hacen más que acrecentar las diferencias de capacidades de investigación entre los centros y las periferias del sistema académico mundial. Las revistas se convierten en instrumentos de consagración que en este contexto logran afincar prácticas que valoran la cantidad (de citas, referencias, artículos, revistas) en detrimento de la calidad de la producción científica.

Como parte neural de estas asimetrías, las bases indexadoras revisten especial importancia. El fenómeno de integración vertical y horizontal, la transnacionalización de las empresas y la concentración del mer-

DADOS - Revista de Ciências Sociais, Rio de Janeiro, vol. 61, nº 1, 2018 
cado en manos de unos pocos consorcios afecta a toda la industria de los contenidos, sean estos libros, revistas o soportes multimediales electrónicos. Lo importante del caso es que estas grandes empresas editoriales son las responsables de las bases y repositorios más "prestigiosos" del planeta. Corporaciones como Reed-Elsevier y Thomson \& Reuters son las responsables de la Web of Science y de Scopus, las dos indexadoras que han logrado en las últimas décadas posicionar sus análisis cienciométricos ${ }^{6} \mathrm{y}$ listados de revistas indexadas como puntos de referencias para la producción científica mundial.

En un artículo reciente Larivière, Haustein y Mongeon (2015) demostraron a partir del análisis de 45 millones de documentos indexados en Web of Science durante 20 años, tanto en ciencias físico-naturales como en ciencias sociales, que sólo cinco grandes corporaciones (ReedElsevier, Wiley-Blackwell, Springer, Taylor \& Francis y Sage) alcanzan una concentración de más del $70 \%$ en algunas disciplinas y más del $50 \%$ tomando el total de publicaciones durante 2013. La conformación oligopólica de la comunicación científica ha llevado a una disociación entre la genuina producción de conocimiento científico y su circulación. La comunicación científica se piensa a partir de su impacto y citación en relación con lo que estas grandes corporaciones comerciales entienden como "excelencia" en detrimento de evaluaciones de la ciencia basadas en la calidad y en el impacto social de su transferencia.

Guédon (2008) destaca que la estratificación de la competencia dentro de la ciencia es una idea compartida dentro de la comunidad científica, pero su transformación progresiva en una estructura oligopólica de poder, muchas veces, es poco cuestionada. Más concretamente, el creciente proceso de mercantilización del conocimiento ha llevado a consolidar grandes niveles de ingresos para las corporaciones editoriales. Si se toman en cuenta las ganancias del período 2012-2013 de las 5 grandes corporaciones oligopólicas de la comunicación científica mundial suman cada una más de 2 billones de dólares: "un $42 \%$ más que empresas como Pfizer, 29\% más que el ICBC y muy por arriba del $10 \%$ de Hyundai Motors, que son las empresas farmacéuticas, bancarias y automotrices más redituables según Forbes' Global 2010" (Larivière, Haustein y Mongeon, 2015:10). El negocio de la comunicación científica claramente trasciende el ámbito académico impregnando el sistema académico mundial con un entramado de acumulación y concentración de capital que impacta directamente en las formas en que la ciencia se comunica y divulga su conocimiento. 
Más Allá de la Indexación: Circuitos de Publicación de Ciencias Sociales...

Ante este contexto problemático del sistema de publicaciones, en las últimas décadas se ha dado lugar a un conjunto de iniciativas tendientes a superar estas asimetrías de la comunicación y divulgación científica $^{7}$. Las luchas por el capital simbólico entre investigadores y editores comerciales profundizan una serie de cuestiones de gran importancia en la configuración actual de poder en el sistema académico. Babini destaca que:

Hace poco se cumplieron 20 años de la Propuesta Subversiva de Stevan Harnad, investigador de ciencias cognitivas y acceso abierto en las universidades de Southampton, Gran Bretaña, y Quebec en Montreal, Canadá. Esta propuesta invitaba a los investigadores a subir a Internet el texto completo de sus artículos publicados y ofrecerlos gratis al público. Un paso más a lo que venían haciendo los físicos, quienes desde 1991 subían sus artículos de investigación en $\operatorname{arXiv}^{8}$, un repositorio digital temático en física, matemáticas y computación que hoy tiene cerca de un millón de textos completos y gratuitos. A inicios de los 2000 se acuñó el concepto acceso abierto para denominar este movimiento internacional hacia la liberalización del acceso a los resultados de investigaciones financiadas con fondos públicos, ofreciendo el acceso al texto completo y datos de investigación, gratis en Internet (Babini, 2014:1).

Diferentes iniciativas internacionales y regionales han abocado sus esfuerzos para potenciar el alcance de las políticas de acceso abierto9 que se han visto sintetizadas en diferentes declaraciones e iniciativas (Declaración de Budapest 2002, Declaración de Berlín 2003, Declaración de Bethesda 2003, Declaración de Salvador 2006).

La presencia de políticas de acceso abierto en América Latina y el Caribe es amplia y se ha convertido en una referencia a nivel mundial. Estudios recientes han avanzado hacia diferentes explicaciones de la expansión del sistema latinoamericano de publicaciones considerando a las políticas de acceso abierto como la principal de ellas (Alperin, Fischman y Willinsky, 2011; Fischman, Alperin, y Willinsky, 2010). La búsqueda por generar espacios gratuitos al acceso de la producción científica ha llevado a cimentar una práctica de edición y publicación de resultados científicos pensada a partir de su consideración como bien común.

Las tensiones entre la comercialización y el acceso abierto, la internacionalización y la búsqueda por generar contribuciones locales, la evaluación cuantitativa y la evaluación de la calidad (originalidad de las

DADOS - Revista de Ciências Sociais, Rio de Janeiro, vol. 61, nº 1, 2018 
revistas y distintas modalidades de referato), entre otras, son fenómenos que inciden directamente sobre las revistas científicas. A partir de estas tensiones, se han desarrollado diferentes articulaciones que han marcado segmentaciones en la circulación del conocimiento científico en América Latina.

\section{Segmentaciones en la circulación de las CSyH vía publicaciones periódicas}

Buena parte de los estudios sobre circulación del conocimiento se centran en el análisis de las publicaciones científicas como parte de un conjunto de articulaciones vinculadas a su acceso, impacto, circulación y alcance. En los últimos años, la obsesión por la indexación y el índice de impacto ha sido promovida directamente desde las políticas científicas nacionales. Discusiones acerca del productivismo y la calidad de las publicaciones han llevado a la generación de diversos posicionamientos que han cuestionado la estratificación y posterior diferenciación en grupos de las revistas evaluadas (Rego, 2014; Bianchetti, 2007, 2010; Machado, 2007; Alcadipani, 2011; Chauí, 1989; Coimbra, 2009; Sguissardi y Silva Júnior, 2009; Vilaca y Palma, 2013; Mugnaini, 2006).

Durante la última década se puede observar un fuerte desarrollo de la comunidad científica brasileña con relación a la expansión de indicadores cienciométricos que responde a la implementación de criterios de evaluación de la ciencia por la Coordinación de Perfeccionamiento de Personal de Nivel Superior (CAPES por su sigla en portugués). Creada en 1976 para evaluar el desempeño de los programas de graduación y pos graduación consolidó ya a partir de 1998 criterios cuantitativos de evaluación ${ }^{10}$. Así es, como el gobierno brasileño creo en 2009 el registro de producción científica QUALIS-CAPES que clasifica las revistas nacionales e internacionales en función de un análisis cienciométrico de su producción segmentando las publicaciones periódicas en 8 categorías. Para ello se crearon 9 comisiones disciplinares que en principio poseen autonomía en la elección de criterios de evaluación de las revistas. Sin embargo, CAPES propone que para las dos categorías más altas (A1 y A2) se tomen en consideración el H-Index, el factor de impacto y otras mediciones de excelencia, así como la inclusión de las publicaciones en Web of Science y Scopus.

Carvalho Neto, Willinsky y Alperin (2016) diseñaron una tabla con la estandarización de los criterios de selección por disciplinas en los es- 
Más Allá de la Indexación: Circuitos de Publicación de Ciencias Sociales...

tratos de Qualis. La máxima categoría (A1) es alcanzada por las revistas médicas si poseen un Factor de impacto igual o mayor a 4, las de biología igual o mayor a 4,3 y las de ciencias sociales si poseen un H-Index igual o mayor a 20 o un factor de impacto igual o mayor a 1. Por su parte, Mugnaini (2006) demuestra tras un extenso análisis de los documentos de áreas de evaluación de QUALIS-CAPES que los criterios de evaluación definidos favorecen a las revistas denominadas "internacionales"11 por el uso del Factor de Impacto en detrimento de la producción brasileña indexada en SciELO.

Por su parte, Miranda y Mugnaini (2013) analizaron el formulario de evaluación de CAPES destacando que la producción intelectual es uno de los cinco componentes del formulario. El peso de las publicaciones científicas varía entre un $30 \%$ y un $65 \%$ teniendo un peso primordial en las evaluaciones el Factor de Impacto (producto del Journal Citation Report de la empresa Thomson Reuters), seguido de la indexación de las revistas en bases como Web of Science o Scopus. Mugnaini (2014) concluye que la relación establecida en Brasil entre indicadores bibliométricos y política científica, tomando estudios cuantitativos de la ciencia como utilidad nacional, produce una excesiva dependencia de los productos y recursos de las grandes empresas científicas como Thomson Reuters o Elsevier.

En el caso argentino, en 2014 el CONICET (Consejo Nacional de Investigaciones Científicas y Técnicas) a través de su resolución nE 2249 del Directorio $^{12}$, promovió la formalización de tres niveles de revistas. El primero de ellos se encuentra conformado por las publicaciones indexadas en Web of Science, SCOPUS, Circ-A y SciELO (CONICET, 2014). A pesar de que la resolución posee carácter consultivo es importante destacar que dentro de la institución se ha iniciado un proceso de evaluación de la producción científica en función a su indexación e índice de impacto. Un estudio llevado a cabo por Beigel (2014) sobre los criterios de evaluación de las publicaciones en las comisiones asesoras del CONICET concluye que existe una tendencia general, transversal a todas las áreas científicas, a priorizar la indexación de las revistas por sobre la evaluación de la originalidad del artículo.

Por otra parte, realizar un análisis de la producción y difusión de las ciencias sociales y las humanidades, basado en los registros de SCOPUS y Web of Science conlleva limitaciones. En principio, el número absoluto y relativo de revistas latinoamericanas indexadas en estas grandes corporaciones académicas es mínimo. Las publicaciones

DADOS - Revista de Ciências Sociais, Rio de Janeiro, vol. 61, nº 1, 2018 
de Estados Unidos, Francia, Inglaterra, Alemania y Japón representan el 78\% del total de los registros incluidos en Scopus y WoS, mientras que las revistas de América Latina y el Caribe alcanzan apenas el 9\%. Teniendo en cuenta una diferenciación por áreas científicas la brecha se acrecienta aún más, la inclusión de revistas de ciencias físico-naturales es inmensamente mayor a las de ciencias sociales y humanidades en estos espacios. Por lo tanto, si los estudios se concentran sobre esta cantidad mínima de revistas, lo que se analiza en definitiva son las publicaciones que han adaptado sus pautas editoriales a los requerimientos "mainstream" del sistema de publicaciones, por lo que sus conclusiones deben relativizarse si se quiere analizar la producción científica de regiones periféricas como Latinoamérica de manera integral.

Si se puntualiza la mirada en los indexadores de corriente principal, existen claras diferencias entre Web of Science (en este caso el Social Science Citation Index -SSCI- y el Arts and Humanities Citation Index -A\&HCI-) y Scopus. El promedio latinoamericano de revistas en WoS es de 0,9 por lo que países como Chile $(2,8 \%)$, México $(1,6)$ y Brasil $(1,2 \%)$ son la punta de lanza de la región. Es destacable el escaso número de revistas argentinas en este indexador $(0,5 \%)$ siendo el segundo país con mayor cantidad de publicaciones periódicas activas de la región. Para el caso de Scopus, la mayoría de los países se acercan al promedio latinoamericano (3,1\%) mientras que nuevamente Argentina se sitúa muy por debajo del mismo $(0,9 \%)$. Brasil y Chile poseen mayor representación que la media en este caso en tanto que Venezuela se suma al podio en el indexador de excelencia de Elsevier. A nivel general, Latindex Catálogo es la base más inclusiva con casi $30 \%$ de revistas seguida lejanamente por RedALyC $(6,8)$ y SciELO $(6,5 \%)$.

Para este trabajo construimos una base de datos con revistas de ciencias sociales y humanidades de Argentina y Brasil activas al año 2015. Dichas revistas fueron caracterizadas en función de su indexación ${ }^{13}$, pertenencia disciplinar ${ }^{14}$, lugar de edición (institución editora) y acceso (soporte, disponibilidad a texto completo, sitio electrónico). En un primer momento, nos abocaremos a analizar las principales características de las revistas en ambos países a partir de la utilización de tres índices de correlación ${ }^{15}$ para profundizar el estudio de las revistas de cada país.

La expansión de la edición de revistas en ambos países puede observarse a mediados de los noventa (Guimarães y Humann, 1995), década en la cual las tecnologías de la información y comunicación tuvieron su 


\section{Gráfico 1}

Revistas de CSyH en Argentina y Brasil por Fecha de Creación (n: 4459)

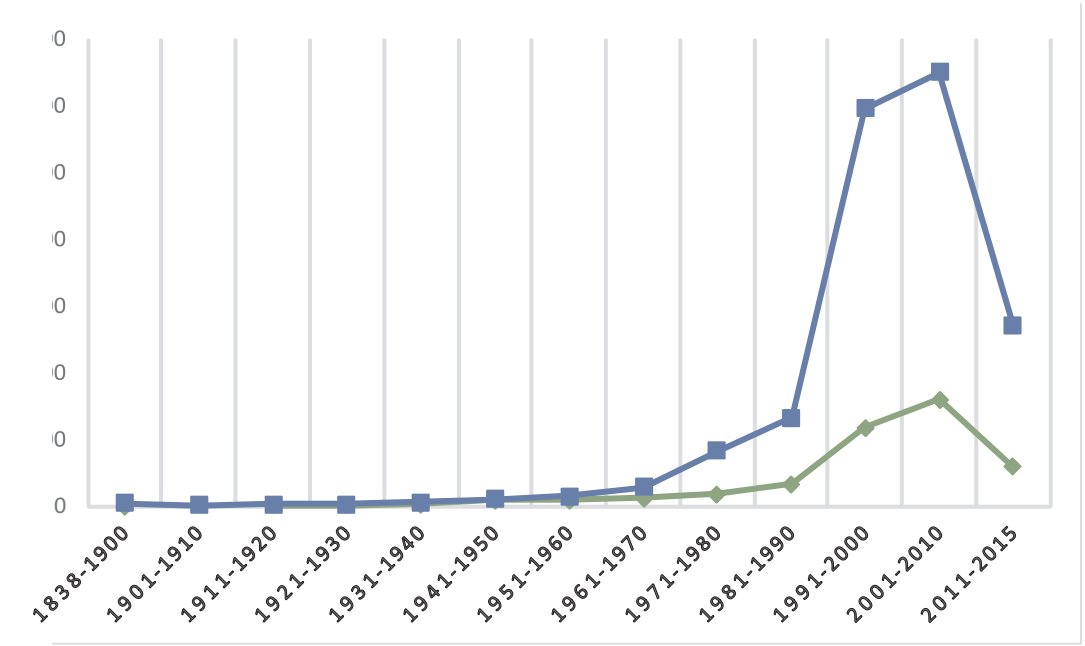

Fuente: Construido por el autor.

mayor impacto en la región. La consolidación de la vía digital como soporte de edición de las publicaciones periódicas es un dato relevante para pensar las últimas tres décadas estudiadas. A partir del año 1997 hasta la actualidad, se edita en algún soporte digital más del 55\% de las publicaciones periódicas relevadas.

En el siguiente gráfico se muestran las revistas activas de CSyH de ambos países por fecha de creación. En la base de estudio se puede observar una tradición de edición de revistas en Argentina a partir de la década de 1940. Al año 1990 se habían editado el 19\% del total de revistas argentinas lo que indica un acelerado crecimiento en los últimos 25 años. Para el caso brasileño, el crecimiento puede identificarse a partir de 1974 hasta su expansión más fuerte alrededor de 1996 (hasta este año sólo se había editado el 25\% del total).

En 1996 se llevó a cabo la Conferencia Anual sobre Publicación Electrónica en Ciencia organizada por la UNESCO en París, donde se abordaron las principales problemáticas de la edición de revistas científicas en países periféricos. En su informe final, Cetto y Voutssas (1996) específicamente sobre América Latina realizaron un diagnóstico de la edición de publicaciones periódicas basándose en CLASE, Periódica y los 
registros de Ulrich's Periodical Directory en donde determinaron la necesidad de introducir las TICs en la gestión editorial y la necesidad de contar con un registro de indexación regional (Cetto y Voutssas, 1996). Su interrogante de partida fue plantear si la expansión de las TICs representaría una mejora en la circulación de la producción latinoamericana a nivel mundial, interrogante que se encuentra aún vigente 20 años después. Como hemos visto con la llegada del nuevo milenio se han creado muchísimas revistas, la mayoría de ellas en formato digital, no obstante, nos queda aún la incertidumbre de conocer qué espacios de circulación y visibilidad poseen.

Si nos situamos a partir del criterio disciplinar, para el caso argentino, más del 70\% de las revistas son disciplinares y de ellas, el 53\% corresponden a disciplinas de ciencias sociales. Existe una importante cantidad de revistas consideradas interdisciplinarias y un escaso número de las denominadas temáticas. Para el caso brasileño, las proporciones son similares, sólo con un ligero aumento de revistas de humanidades

\section{Gráfico 2}

Principales Instituciones Editoras de Revistas de CSyH

(Argentina y Brasil)

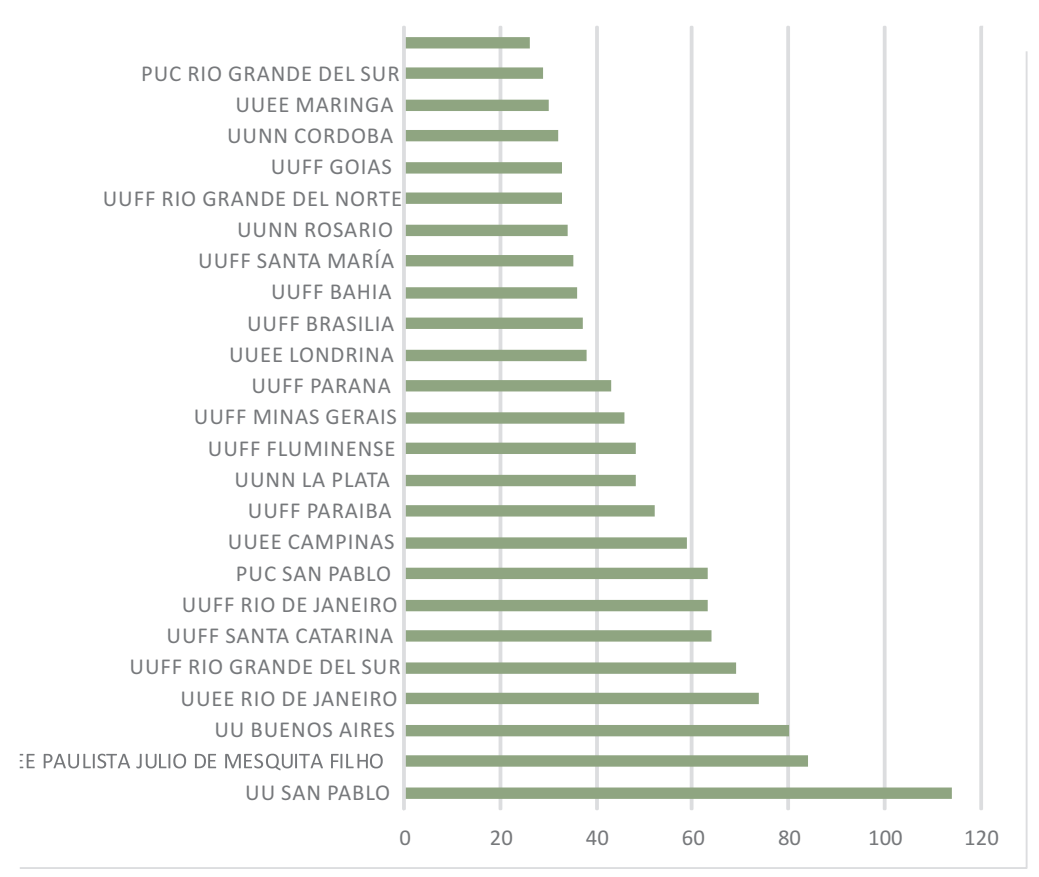

Fuente: Construida por el autor. 
Más Allá de la Indexación: Circuitos de Publicación de Ciencias Sociales...

y una disminución al 44\% de ciencias sociales. Es claro el predomino de disciplinas como psicología, educación, derecho y economía en Brasil, sumando entre ellas el 52\% del total de revistas editadas en ese país. Para el caso argentino, disciplinas como historia, literatura, ciencia política y sociología constituyen el $40 \%$ del total.

Ahora bien, si se pone el foco en las instituciones editoras y los lugares de edición de las revistas el análisis se refina un poco más. La mayoría de las revistas se encuentran financiadas por sus respectivos estados nacionales a partir de programas, proyectos y presupuestos anuales regulares ya que forman parte de instituciones de educación superior estatales. La estructura de la comunicación científica en América Latina es eminentemente pública por lo que son las universidades nacionales, estaduales y federales (para el caso argentino-brasileño) las principales responsables de su funcionamiento. A continuación, se grafica la distribución en términos absolutos de las 25 instituciones editoras con mayor cantidad de revistas editadas.

La Universidad de San Pablo, la Universidad Estadual Paulista, la Universidad de Buenos Aires, la Universidad Estadual de Río de Janeiro y la Universidad Federal de Río Grande del Sur son las cinco instituciones editoras más importante. Si agrupamos las instituciones editoras por provincias o regiones tanto San Pablo y Río de Janeiro para el caso brasileño como la Ciudad Autónoma de Buenos Aires y la Provincia de

Gráfico 3

Circuitos de Publicación de CSyH en Argentina y Brasil (n: 4459)

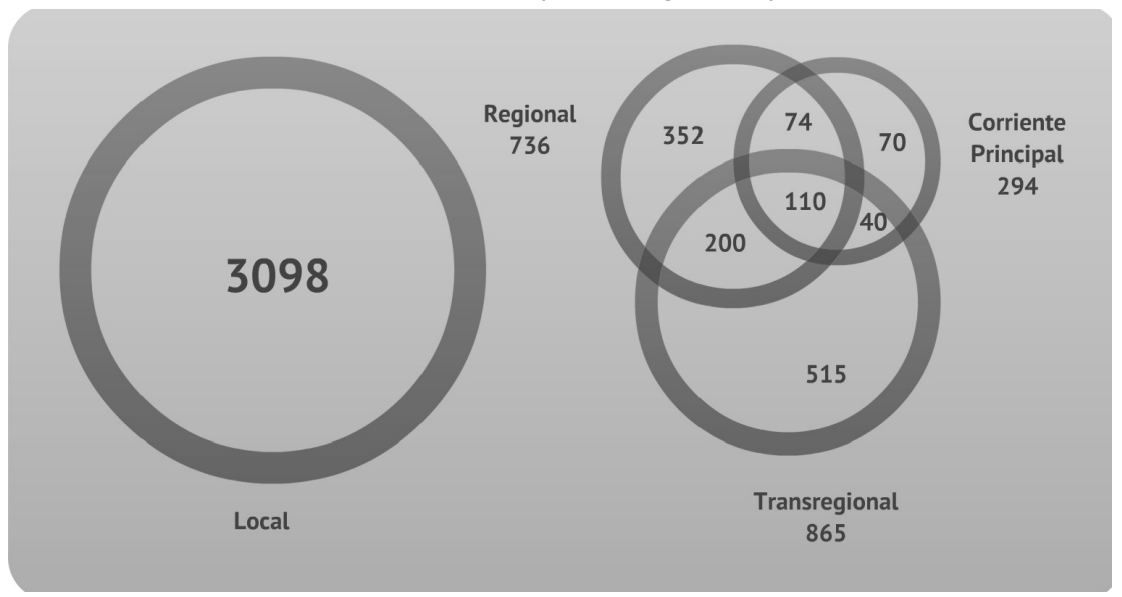

Fuente: Construida por el autor.

DADOS - Revista de Ciências Sociais, Rio de Janeiro, vol. 61, nº 1, 2018 
Buenos Aires para el caso argentino son el centro neurálgico de la producción de publicaciones periódicas. En ellas se concentra más del $47 \%$ de las revistas. En este sentido, Argentina posee un mayor grado de concentración en tanto que en Brasil estados como el de Río Grande del Sur y Minas Gerais poseen una presencia importante.

\section{CIRCUITOS DE PUBLICACIÓN DE CSYH EN ARGENTINA Y BRASIL}

Las revistas analizadas en este trabajo se vinculan con cuatro circuitos de publicación: mainstream o de corriente principal, transregional de acceso abierto, regional y local. Hacia el interior de cada uno de ellos, se insertan revistas similares con pautas editoriales comunes pero que, debido a los cambios en la comunicación científica, al mismo tiempo, han desarrollado múltiples modalidades de circulación. Esto se puede observar en la existencia de yuxtaposiciones entre circuitos

La preeminencia del circuito local nos obliga a repensar la manera en que consideramos al mundo editorial de revistas en Argentina y Brasil. Si anteriormente mencionábamos las limitaciones de referirnos a las publicaciones periódicas latinoamericanas a partir de un análisis basado en WoS o Scopus, podemos postular lo mismo con relación a SciELO, Latindex Catálogo o RedALyC. Casi el 70\% de las revistas estudiadas no se encuentran incluidas en ningún indexador regional, transregional de acceso abierto o de corriente principal. Por tanto, consideramos que profundizar el análisis en este espacio local de circulación nos puede brindar un panorama más integral de la circulación de conocimiento científico vía revistas en estas dos potencias regionales. Con el fin de caracterizar más profundamente las revistas de cada país proponemos a continuación un análisis de correlación basado en tres coeficientes para examinar el mundo de las $\mathrm{CSyH}$ publicadas en revistas en dos casos nacionales.

\section{Las Revistas que Participan del Circuito Regional}

Una cuestión fundamental para analizar la circulación de revistas en la región se encuentra determinada por el acceso a las mismas. La visibilidad se manifiesta como un factor que logra generar espacios de comunicación científica tan importantes como la indexación. En este sentido, los proyectos como RedALyC, SciELO y Latindex Recurso Electrónico lograron generar posibilidades de acceso a artículos a texto 
Más Allá de la Indexación: Circuitos de Publicación de Ciencias Sociales...

completo cuando indexadores cerrados como Web of Science o Scopus poseen un circuito comercial de acceso cerrado altamente oneroso.

La posibilidad de acceso irrestricto a las revistas científicas y sus contenidos ha sido la punta de lanza de todo un movimiento mundial dedicado a pregonar el acceso abierto y la democratización de la ciencia a partir de una fuerte crítica a la creciente mercantilización del conocimiento científico. En este sentido, tanto el agrupamiento de revistas del circuito regional como especialmente aquellas que pertenecen al circuito transnacional de acceso abierto son un fiel reflejo de la sustanciación de estas iniciativas a niveles editoriales. Para este caso la categoría a texto completo en SciELO (test-value 18,71) es la más relevante seguida por la categoría Recurso Electrónico de Latindex (test-value 6,67 ). SciELO es una plataforma que permite el acceso a contenidos (revistas y artículos) de manera gratuita y sin suscripción previa. Todas sus colecciones se encuentran disponibles mientras que el "Recurso Electrónico" de Latindex es un producto diferente (aunque no excluyente) del Catálogo y del Directorio por lo que posee una dimensión sustantivamente menor.

Una consideración con relación al acceso y posterior utilización de las revistas tiene que ver con el idioma de la publicación. La inclusión de revistas latinoamericanas en bases regionales refiere a espacios de circulación en español y portugués mayoritariamente. Cuestión no menor si consideramos que indexadores del tipo Scopus o WoS son recursos en inglés con todas las limitaciones que eso implica para regiones periféricas no angloparlantes como la nuestra.

El circuito regional está compuesto por 736 revistas. De las cuales, el $54 \%$ corresponden a revistas brasileñas y el $46 \%$ a revistas argentinas. Este dato debe ser analizado con un poco más de detenimiento. Las 399 revistas brasileñas de este espacio representan un $11 \%$ del total de revistas de ese país, mientras que las 377 revistas argentinas representan un $44 \%$ del total. Esto nos demuestra el papel que la circulación regional posee para las revistas argentinas y la necesidad de matizar la presencia brasileña en este circuito.

El circuito regional posee varias intersecciones con los demás espacios. Más de la mitad de las revistas incluidas allí se encuentran insertas en el circuito de corriente principal o en el circuito transnacional de acceso abierto. Esto responde a un creciente proceso de los últimos años de diversificación de la circulación de las revistas en función de las posibi- 
lidades de acceso y las trampas de la indexación. Si la inclusión de una revista en Scopus representa la imposibilidad de acceder a su contenido de manera gratuita, su indexación en SciELO le permite tener visibilidad a nivel regional y a texto completo. Tal es el caso de Dados editada por la Universidad Estadual de Río de Janeiro que se encuentra indexada en el Social Science Citation Index pero al mismo tiempo se encuentra alojada a texto completo en el portal SciELO; en la misma situación se encuentran un grupo de revistas como por ejemplo: Ciencia \& Salud Colectiva (Asociación Brasileña de Posgraduación en Salud Colectiva), Transinformación (Pontificia Universidad Católica de Campinas), Intersecciones en Antropología (Universidad Nacional del Centro de la Provincia de Buenos Aires), Tiempo Social (Universidad de San Pablo), entre otras.

Con relación a la presencia disciplinar, las revistas de historia, las interdisciplinarias de las ciencias sociales y la sociología son las categorías que más inciden en este espacio.

Si consideramos la fecha de creación, el circuito regional se encuentra nutrido de revistas que han sido fundadas en las décadas de los setenta y ochenta. Décadas previas a la gran expansión de la edición en la región lo que demuestra una práctica editorial que se ha consolidado en el tiempo (por lo menos 25 años) y que ha sabido adaptarse a las nuevas plataformas de la comunicación científica. Esta apreciación se comprueba con la presencia de la categoría de soporte papel y digital en este circuito (test-value 12,81). Estas son revistas que iniciaron su publicación en papel y que adoptaron al mismo tiempo un soporte digital, preservando su circulación hasta la actualidad.

\section{Las Revistas del Circuito de Corriente Principal}

El marco distintivo del circuito de corriente principal se encuentra vinculado a los medios de acceso y visibilidad de la producción incluida en el mismo. En este caso, su indexación en corporaciones como Web of Science o Scopus trae consigo la imposibilidad de acceder a esas revistas de manera gratuita y a texto completo. Además, un grupo importante de estas revistas poseen modalidades híbridas de acceso abierto (APC - Author Proccesing Charges -, mandatos largos de archivo y licencias de circulación restrictiva) en sus políticas editoriales lo que profundiza los problemas de visibilidad y acceso de este grupo. Este circuito forma parte de una estructura internacional que a partir de la creación del Science Citation Index-SCI logró privilegiar instrumentos 
Más Allá de la Indexación: Circuitos de Publicación de Ciencias Sociales...

de evaluación y circulación de la ciencia basada en parámetros de "excelencia" (Guédon, 2001; Gingras, 2002; Beigel, 2014; Ortiz, 2009). Esta configuración construyó una jerarquización de la producción científica que fue descripta anteriormente y que dio sustento a la constitución actual del sistema académico mundial.

Casi el 70\% de este circuito está compuesto por revistas brasileñas. Esta conformación del circuito responde a una apuesta muy fuerte por parte del estado brasileño de potenciar sus revistas científicas (vía financiamiento) en búsqueda de situar su producción en los centros del SAM. Muchas de las políticas llevadas a cabo por CAPES (Guimarães y Humann, 1995), FAPESP y otras organizaciones lograron generar las condiciones necesarias para la modernización de la gestión editorial. La búsqueda por la indexación en bases prestigiosas (WoS, Scopus, Recursos de ProQuest, EBSCO, entre otros) fue además dinamizada por los requerimientos de una cultura evaluativa heterónoma implementada por CAPES a los programas de posgraduación.

Existe en este espacio una fuerte presencia de revistas de psicología (test value 2,71), lingüística (test value 2,63), economía (test value 2,55) y de estudios de salud colectiva (test value 2,31). Revistas como el Journal of Applied Economics, Revista Brasileña de Psiquiatría, Revistas Argentina de Clínica Psicológica, Revista de Administración de Empresas y Psicología: Teoría e Investigación, son ejemplos de publicaciones periódicas disciplinares que forman parte del selecto grupo de revistas indexadas en la corriente principal.

En términos relativos, el circuito mainstream se encuentra compuesto por revistas editadas en instituciones de diversas características. La presencia de Fundaciones y Universidades Confesionales es muy importante, así de universidades públicas como la Universidad Estadual Paulista, la Universidad Federal de Río Grande del Norte y la Universidad Estadual de Campinas. Las fundaciones CESGRANRIO, Oswaldo Cruz y Getulio Vargas, como las universidades PUC Río de Janeiro y la Universidad de Vale do Rio dos Sinos poseen pocas publicaciones, pero la mayoría de ellas se encuentran indexadas en el Social Science Citation Index o en Scopus.

\section{Las Revistas Insertas en el Circuito Trasnacional en Acceso Abierto}

El denominado circuito transnacional de acceso abierto agrupa revistas que poseen casi la totalidad de sus recursos en códigos abiertos.

DADOS - Revista de Ciências Sociais, Rio de Janeiro, vol. 61, no 1, 2018 
Mayormente estas revistas fueron creadas durante la primera década del siglo XXI. Desde la publicación en formatos y soportes artesanales del tipo HTML a la llegada de plataformas online de publicación tales como OJS-PKP o Scholar One, estas revistas se encuentran agrupadas en directorios como DOAJ, DIALNET y Ulrich principalmente, así como también en repositorios regionales como LA-Referencia o CLACSO. La categoría digital de soporte en este circuito posee un test-value de 14,89, el más representativo de todos los circuitos. Esto marca un contrapunto con la gran producción de CSyH que sólo se edita en papel. La distribución y circulación vía un medio digital (desde la carga a la web de un archivo en PDF hasta la adopción de un software de gestión editorial del estilo OJS) habilita la posibilidad de buscar una revista y todo su contenido solamente contando con acceso a internet. La visibilidad de los medios digitales ha ayudado a difundir y posicionar las revistas a escalas que sobrepasan sus límites institucionales (políticos, económicos y de logística). No obstante, es importante aclarar, que la visibilidad no indica necesariamente el acceso o posterior utilización de esos contenidos (ya sean usos académicos o de transferencia concreta a una determinada comunidad). La inclusión de una revista en DOAJ aumenta sus posibilidades de circulación concreta ya que cuenta con el respaldo de una institución reconocida a nivel global que cuenta con filtros, herramientas de búsqueda, información detallada del tipo de contenido, etc.

Como hemos mencionado anteriormente, el acceso abierto es un fenómeno que posee muchas aristas y dimensiones. Una cuestión de gran relevancia es la disponibilidad a texto completo en dominios de acceso abierto. No todos los portales, bases, indexadores o repositorios que se encuentran en acceso abierto garantizan la posibilidad de acceder a todos los contenidos de las revistas (tanto a los números más recientes como a los más antiguos). Las categorías a texto completo SciELO (test value 8,13$)$, OJS-PKP $(6,19)$ y Recurso Electrónico de Latindex $(5,61)$ se sitúan como indicadores potentes en la explicación de este circuito.

Es importante destacar el elevado número de revistas que exclusivamente se encuentran insertas en este espacio (casi el 60\%). De este agrupamiento el $82 \%$ son revistas brasileñas que se hayan mayoritariamente incluidas en DOAJ. El directorio de revistas en acceso abierto (DOAJ por su acrónimo en inglés) fue uno de los primeros sitios a nivel mundial que postuló los mandatos del acceso abierto. Se inició como proyecto en 2003 en la Universidad sueca de Lund con 300 revistas, 
Más Allá de la Indexación: Circuitos de Publicación de Ciencias Sociales...

actualmente cuenta con 9.349 revistas y 2.345 .538 artículos de más de 128 países.

Con relación a la infraestructura institucional del circuito, existe un gran y diverso grupo de instituciones editoras que poseen sus publicaciones insertas en este circuito. Tal es el caso de la Universidad Nacional de la Plata, Universidad Estadual de Maringá o de la Pontifica Universidad Católica de Buenos Aires con revistas de relevancia y gran circulación como Sapientia, Synthesis, Mundo Agrario, Diálogos o Acta Scientiarum.

\section{Los Circuitos Locales}

El último de los agrupamientos es el circuito que hemos denominado como local. Este circuito se encuentra compuesto por revistas que son editadas mayormente en formato papel (test-value 23.5) o en formato digital sin texto completo, por lo que poseen una distribución restringida.

El dato más relevante de este circuito es que representa casi el 70\% del total de revistas de la base estudiada. Teniendo en consideración que este circuito es el único que puede ser completamente englobado como tal (ya que existen yuxtaposiciones entre los circuitos regionales, de corriente principal y transnacional) la proporción es muy alta y representa un claro ejemplo de las limitaciones de circulación y visibilidad de las revistas de la región.

Este circuito se encuentra compuesto en su mayoría por revistas editadas en Brasil. Hacia su interior se destacan revistas que fueron creadas recientemente (primera década del siglo XXI) o en la década de los noventa. Las revistas de fundación más antigua son principalmente boletines y cuadernos de departamentos, institutos universitarios o cátedras mientras que las revistas recientemente creadas poseen características, tales como su publicación en soporte digital o adecuación a criterios editoriales formalizados, que permiten considerarlas como en vías de indexación.

Hacia el interior del circuito local, se puede observar en ambos países una fuerte presencia de las artes y humanidades en relación con otros circuitos. El eje fundamental en este circuito se centra en la presencia absoluta y relativa de revistas brasileñas (más del $85 \%$ ). La mayoría de ellas son revistas multidisciplinarias (test-value 14,16) y jurídicas

DADOS - Revista de Ciências Sociais, Rio de Janeiro, vol. 61, nº 1, 2018 
(test-value 6,29). La presencia como líder regional científico de Brasil es indiscutida (en muchas áreas científicas, en infraestructura, en formación de recursos humanos, en inversión en ciencia y tecnología, en producción científica) sin embargo, la constitución de un circuito eminentemente local de circulación de sus revistas de CSyH amerita una reflexión más profunda acerca de la concreta incidencia e impacto de su producción.

Si nos situamos a partir de las instituciones editoras, universidades prestigiosas de gran presupuesto y reconocidas internacionalmente tales como la Universidad de San Pablo y la Universidad de Buenos Aires, editan un gran número de publicaciones, sin embargo, gran parte de sus producciones se encuentran insertas en el circuito local. La USP posee un $48 \%$ de sus revisas en este circuito, la UBA un $42 \%$, la Universidad Estadual Paulista (UNESP) un 59\%, la UERJ un 58\% y la UFRJ un $64 \%$.

Las editoriales comerciales representan un 4,5\% del total de revistas estudiadas contando en el circuito local con una mayor representación (test-value 6,61). Este porcentaje es un contrapunto importante con la expansión de la edición comercial de revistas en los centros del sistema académico mundial $\mathrm{y}$, por tanto, un dato importante para pensar la gestión editorial en la región.

Las consideraciones referidas al circuito local no buscan desmerecer las revistas que allí se incluyen. Muchos menos ponderarlas negativamente con relación a la población de revistas indexadas estudiadas. Simplemente nos preguntamos acerca de los motivos por los cuales hay tantas revistas no incluidas en bases o repositorios regionales o transnacionales en acceso abierto. Desde Latindex Catálogo hasta SciELO, América Latina cuenta con amplios espacios de inclusión de la producción científica en bases con características no tan restrictivas y onerosamente inviables como las de corriente principal. Asimismo, es valedero preguntarse qué tipo de resultados de investigaciones circulan en este espacio, qué temas y qué problemáticas teóricas y empíricas.

Como hipótesis de trabajo y en desarrollo, planteamos que este fenómeno se debe, principalmente, a la transformación de la función primigenia de las revistas científicas; de ser una vía de comunicación a erigirse como una de los principales instrumentos de consagración. Este efecto de concentración y acumulación de capital simbólico de las 
Más Allá de la Indexación: Circuitos de Publicación de Ciencias Sociales...

revistas en los espacios nacionales ha tenido consecuencias políticoinstitucionales.

La creación de revistas sin una política editorial definida, fundada por los intereses de una red o grupo de investigación, llevadas adelante por académicos sin formación editorial, pensadas a partir del posicionamiento de sus colaboradores en el marco de su institución editora, con evaluaciones endogámicas, compuestas por artículos recibidos por "invitación", entre otras causas, implica la estructuración de revistas débiles, de corto alcance, con circulación restringida y con problemas serios de distribución. Por otra parte, son revistas con una incidencia intramuros de relevancia ya que sostienen la práctica de publicación a escala institucional lo que permite una plataforma de circulación orientada nacionalmente. En este sentido, estas revistas forman parte de estrategias de consagración local por fuera de las reglas de la evaluación cuantitativa de la ciencia.

\section{CONCLUSIONES}

A la hora de referirse al prestigio o importancia de una publicación se suele mencionar como correlato dónde se encuentra indexada dicha revista. Es una relación de sentido común dentro del mundo académico ya que forma parte de un habitus que atraviesa la práctica científica y que modela los diferentes procesos de desarrollo de la ciencia, sus resultados y finalmente, su comunicación. Mencionar que tal revista forma parte de Web of Science o que se encuentra disponible en Scopus es sinónimo de "excelencia" y, por carácter transitivo, de "calidad".

En este trabajo hemos llevado a cabo un análisis empírico con el fin de demostrar que en última instancia el velo de la indexación refleja un mecanismo de jerarquización de las revistas construido localmente a partir de premisas globales. La indexación puede ser considerada como un punto de partida, pero es necesario complejizar el análisis con la intervención de otros elementos analíticos para poder generar un diagnóstico de la circulación de revistas de CSyH en Argentina y Brasil. En un estudio reciente se observa: "un elevado nivel de similitud entre los criterios utilizados por las evaluaciones de Latindex, RedALyC y SciELO, de las 15 categorías, 12 son unánimes" (Oliveira Morin et al., 2014:72). Dichos criterios de evaluación son los considerados para la inclusión o no de las publicaciones periódicas en estos indexadores regionales por lo que finalmente, y más allá del marketing

DADOS - Revista de Ciências Sociais, Rio de Janeiro, vol. 61, no 1, 2018 
científico de cada uno de ellos, se asemejan bastante en sus prácticas de "gatekeepers" de la ciencia.

A lo largo del trabajo se destacó el papel de las revistas a la hora de pensar la comunicación científica y su rol en la evaluación de científicos e instituciones. En este sentido, y con el objetivo de indagar más allá de una mirada sobre la visibilidad, el acceso y la indexación, se propuso la idea de "circuito de publicación" para pensar y repensar las formas en que las publicaciones periódicas se convierten en vehículos de prestigio y consagración.

Cada uno de los circuitos construidos poseen características particulares $\mathrm{y}$, por tanto, hacen referencia a una determinada forma y práctica científica que se sustancia en cuerpos editoriales, estrategias de publicación por parte de los autores, mecanismos de evaluación por parte de los sistemas científicos nacionales y parámetros de consagración vinculados a criterios cuantitativos. En este sentido, los circuitos de publicación diagraman una red de relaciones en donde el capital simbólico y las diferentes formas de acumulación/concentración del mismo se ven reflejados en una infraestructura político-institucional.

Las revistas de CSyH en Argentina y Brasil se insertan en cuatro circuitos de publicación: regional, transregional, de corriente principal y local. Una primera conclusión es la fuerte segmentación entre las revistas incluidas en el circuito local. Este último representa casi el 70\% del total, lo que implica una fuerte división entre la circulación localnacional de estas revistas con relación al resto más regionalizadas y hasta internacionalizadas. La mayoría de las revistas que componen este agrupamiento son brasileñas y de disciplinas vinculadas a las artes y humanidades. El circuito regional se encuentra vigorosamente promovido por la presencia de Latindex en la región y representa el $55 \%$ del total de revistas indexadas (incluyendo las yuxtaposiciones entre circuitos). Alineado a las estrategias de visibilización regional, el circuito transnacional se compone de revistas que buscan insertarse en las redes globales de acceso abierto. Por último, el circuito de corriente principal se encuentra compuesto por revistas mayoritariamente brasileñas, fuertemente disciplinares y de acceso restringido.

En el contexto de la creciente internacionalización de la ciencia, tanto en Argentina como en Brasil, se adoptaron una serie de criterios de valoración y ponderación de la práctica científica que tendieron a profundizar las desigualdades y asimetrías del sistema. Por un lado, un 
Más Allá de la Indexación: Circuitos de Publicación de Ciencias Sociales...

grupo pequeño de revistas (y con ello se hace referencia a la institución editora, al editor y al cuerpo editorial) lograron generar las condiciones materiales y simbólicas necesarias para ser incluidas en el circuito de corriente principal y, por otra parte, un gran número de publicaciones se encuentran insertas en el circuito local, editados mayormente en papel y con escasa circulación. La visibilidad de las revistas que circulan en ambos circuitos en la región es claramente reducida. Para acceder a las revistas incluidas en la corriente principal se debe pagar altísimas suscripciones mientras que las revistas "locales" ven reducida su circulación por estar editadas mayoritariamente en papel. La pregunta concreta frente a este fenómeno es si existe una diferencia real de calidad, relevancia y de desarrollo científico entre los dos grupos, o si simplemente, estas asimetrías son el reflejo de tensiones vinculadas con diferentes capacidades político-institucionales.

En esta misma línea de reflexión, consideramos que debemos avanzar más allá de la indexación para pensar la circulación del conocimiento científico vía publicaciones periódicas. Es importante volver a situar a la calidad y originalidad de los artículos en el centro de las discusiones referidas al acceso, visibilidad e impacto de las revistas con el fin de evitar la asociación directa existente entre "indexación" y "excelencia". Lo que no implica quedar fuera de la "gran conversación" o reducir los debates a temas "locales o regionales", todo lo contrario, implica generar espacios de valoración y discusión cada vez más abiertos, inclusivos y democráticos.

(Recebido para publicação em 13 de Abril de 2016)

(Reapresentado em 16 de Dezembro de 2016 e em 2 de Fevereiro de 2018)

(Aprovado em 6 de Fevereiro de 2018)

DADOS - Revista de Ciências Sociais, Rio de Janeiro, vol. 61, nº 1, 2018 


\section{NOTAS}

1. Son los escritos de Oscar Varsavsky, Amílcar Herrera y Jorge Sabato en Argentina; José Leite López, Darcy Riveiro y Helio Jaguaribe en Brasil; Miguel Wionczek, Luisa Leal y Alejandro Nadal Egea en México; Francisco Sagasti en Perú; Máximo Halty Carrere en Uruguay; Osvaldo Sunkel en Chile, Marcel Roche en Venezuela, entre otros, los pioneros en estas temáticas.

2. Actualmente, Clarivate que ofrece 2 productos independientes: Web of Science (con más de 54 recursos indexadores y cienciométricos) y Journal Citation Report encargado de generar el Índice de Impacto (IF).

3. Una dificultad importante a la hora de analizar las revistas como objeto de estudio es la imposibilidad de conocer con precisión el total de revistas académicas editadas en la región. A pesar de la existencia de bases de datos regionales, internacionales y en algunos casos nacionales, es aún complicado saber a ciencia cierta el número total en términos absolutos. La superposición entre diferentes listados y bases de datos es amplia y los errores en los registros son frecuentes. Teniendo en consideración los listados de ISSN a finales de la década de 1990 Latinoamérica contaba con 15.049 registros activos. Si consideramos los registros en Latindex Directorio para el año 2014 contaba con 23.987 publicaciones. Actualmente, el autor lleva a cabo un estudio de mayor envergadura con registros de 26 países de América Latina y el Caribe en el que se han corroborado la existencia de 10032 publicaciones periódicas activas al 2015 de todas las disciplinas científicas.

4. Latindex Directorio y Catálogo son recursos de la UNAM creados en 1995 y 1997 respectivamente. Latindex es considerado como el "Portal de Portales" de la región ya que fue uno de los proyectos pioneros de visibilización de las revistas latinoamericanas. SciELO surgió en 1998 como parte de un arreglo interinstitucional entre BIREME, OMS y FAPESP en Brasil; actualmente opera a través de centros nacionales de recopilación de revistas científicas que son evaluadas a partir de un conjunto de criterios de evaluación comunes. RedALyC fue creada más recientemente (2003) en la UAEM con revistas de ciencias sociales y humanidades hasta incluir en la actualidad todas las áreas científicas. RedALyC junto con SciELO ofrecen análisis a nivel de citación y artículo mientras que Latindex sólo funciona a nivel de publicaciones periódicas. Estas tres bases son las representantes más importantes de la región de la vía dorada del Acceso Abierto. Por su parte, Web of Science forma parte de la corporación Thomson \& Reuters y es considerada como la base de datos más prestigiosa del mundo. Scopus, por su parte, forma parte actualmente de la transnacional Reed-Elsevier de capitales holandeses. Surgió como una alternativa a WoS y tiene en su ámbito de acción una serie importante de recursos indexadores y de citación. Ulrich's Directory es un directorio de publicaciones que posee una gran extensión y que fue recientemente comprada por la empresa Pro-Quest. Tanto WoS, Scopus y Ulrich's Directory son bases e indexadores de acceso cerrado e irrestricto, altamente oneroso y gestionados en inglés. DOAJ es considerada la base en acceso abierto más importante del mundo. Fue un proyecto pionero de la Universidad de Leiden y cuanta actualmente con más de 9.000 revistas a texto completo. Véase: Alperin (2014).

5. La cienciometría surgió a finales de la década de 1960 como una especialización de los estudios bibliométricos basados en la aplicación de métodos matemáticos y estadísticos. Existen actualmente muchos debates en torno a la adjudicación y propiedad 
Más Allá de la Indexación: Circuitos de Publicación de Ciencias Sociales...

del término "cienciometría" (Wouters, 1999; Hood y Wilson, 2001; Mingers y Leydesdorff, 2015), sin embargo, se reconoce que su formulación fue realizada por el investigador ruso Vassily V. Nalimov en 1969. Su preminencia a escala global tuvo su anclaje en los proyectos de Eugene Garfield en el Institute for Scientific Information (ISI-WoS) a partir de 1959.

6. Hacemos referencia al tipo de estudios cienciométricos que se basan en datos provenientes de bases e indexadores de corriente principal en inglés y con lógicas político-institucionales muy diferentes a la de regiones periféricas como América Latina y el Caribe.

7. Como una forma alternativa al modelo oligopólico editorial y sus mecanismos de evaluación de la ciencia se han propuesto una serie de mecanismos alternativos, entre ellos, el proyecto altmetrics. Véase: Torres, D., Cabezas, Á. \& Jiménez, E. (2013). Altmetrics: nuevos indicadores para la comunicación científica en la Web 2.0, Comunicar, 41, 53-60. Alperín, J.P. (2015). The Public Impact of Latin America's Approach to Open Access. Tesis Doctoral, Universidad de Stanford. Alperin, J. P. (2013). Ask not what altmetrics can do for you, but what altmetrics can do for developing countries. Bulletin of the American Society for Information Science and Technology, 39(4), $18-21$.

8. Recientemente se ha creado la versión para ciencias sociales y humanidades de arXiv denominada SocArXiv. Véase: https://osf.io/preprints/socarxiv

9. Existen diferentes modalidades de aplicación de los mandatos y políticas de acceso abierto, las más comunes son la vía dorada (revistas de acceso abierto), vía verde (auto-depósito por parte de los autores), vía azul (las agencias de financiamiento obligan al auto-depósito) y una última denominada vía híbrida donde se encuentran aquellas revistas que se están disponibles en acceso abierto pero que los autores deben pagar para publicar o deben acceder a un período de embargo. Sobre los actuales problemas de las modalidades de las revistas híbridas y el APC (Author Proccesing Charges) véase: Babini, Dominique (2014). El riesgo de que el acceso abierto sea integrado dentro del sistema tradicional de publicación comercial - necesidad de un sistema global no comercial de comunicaciones académicas y científicas. RECIIS - Rev Eletron de Comun Inf Inov Saúde. 2014 out-dez; 8(4): 433-437. Un caso particular es el proyecto Sci-hub creado en 2011 por Alexandra Elbakyan que se ha convertido en el sitio web con mayor cantidad de descargas del mundo. En un reciente artículo en Nature se estima que el sitio resguarda más de 50 millones de artículos que se pueden descargar a texto completo y de manera gratuita sólo contando con la dirección URL, PMID o DOI. Véase: http://www.sciencemag.org/news/2016/04/whosdownloading-pirated-papers-everyone

10. El gobierno brasileño formuló, en la década de 1990, políticas públicas de apoyo a la comunicación científica a través de fondos de financiamiento para la gestión y modernización editorial, así como a través de inversión para el acceso a bases de corriente principal. Brasil destinó para el financiamiento del sitio "Portal de Periódicos CAPES" en 2012 R \$ 174.641.010 (alrededor de USD 50.620.582) hasta alcanzar en 2015 el monto de R\$275.937.377 (alrededor de USD 79,981.848) Véase: http:/ / www.capes.gov.br/images / stories / download / sobre / 08042016-ORCAMENTO2004-2015.pdf. Nascimento y Mugnaini (2016). Qualidade de revistas científicas: um estudo da literatura publicada entre 2003 e 2013. XVII Encontro Nacional de Pesquisa em Ciência da Informação (XVII ENANCIB). Salvador de Bahía

DADOS - Revista de Ciências Sociais, Rio de Janeiro, vol. 61, nº 1, 2018 
11. Existe una diferenciación por parte de algunos estudios entre revistas "internacionales" y "nacionales". Las primeras son las editadas en los centros del SAM, en inglés, con altos índices de citación e incluso insertas en bases del tipo WoS o Scopus. Por su parte, las revistas nacionales son aquellas de circulación local y regional, editadas en español o portugués, con índices de impacto nulos o muy bajos. Guédon (2013) destaca que esta división forma parte de una estrategia global de diferenciación entre ciencia principal y ciencia periférica.

12. Para el caso de las ciencias sociales y humanidades.

13. La indexación tiene que ver con la inclusión de las revistas en alguna de las bases o repositorios regionales e internacionales. Dicha inclusión se encuentra supeditada a un proceso de evaluación basado en parámetros y criterios específicos propuestos por las mismas bases indexadoras. Existen bases con criterios muy restrictivos de inclusión hasta bases que sólo evalúan cuestiones formales y editoriales.

14. La caracterización disciplinar de las revistas se basó en los descriptores registrados en las bases y repositorios donde se encuentran alojadas; en los casos de revistas sin indexación se hizo un seguimiento individual en sus sitios web oficiales o en la de sus instituciones editoras. Con el fin de alcanzar una exploración analítica más profunda se han clasificado las revistas como disciplinares (de las ciencias sociales y las humanidades), interdisciplinarias y temáticas. Nos referimos con revistas temáticas a aquellas que poseen un tema u objeto de estudio específico, tal como, estudios de género o estudios del trabajo, y por tanto no pueden ser incluidas en un área disciplinar específica.

15. Se llevó a cabo un análisis de tres coeficientes de correlación (T de Schuprow, V de Cramer y Ji-Cuadrado) el cual fue sintetizado en un índice común denominado test-value. El procedimiento estadístico se realizó a través de la herramienta-Caracterización de una variable cualitativa- mediante el uso del software Coheris Analytics SPAD 8.3. 
Más Allá de la Indexación: Circuitos de Publicación de Ciencias Sociales...

\section{REFERENCIAS}

ALATAS, Syed Farid. (2003), "Academic Dependency and the Global Division of Labour in the Social Sciences". Current Sociology, vol. 51, no 6, pp. 599-613.

(2014), "La Dependencia Académica: El Desafío Intelectual", en F. Beigel; H. Sabea (orgs.), Dependencia Académica y Profesionalización en el Sur: Perspectivas desde la Periferia. Mendoza, EDIUNC.

ALCADIPANI, Rafael. (2011), "Resistir ao Produtivismo: Uma Ode à Perturbação Acadêmica". Cadernos EBAPE.BR, vol. 9, no 4, pp. 1175-1178.

ALPERIN, Juan Pablo. (2013), “Ask Not What Altmetrics Can Do for You, but What Altmetrics Can Do for Developing Countries". Bulletin of the American Society for Information Science and Technology, vol. 39, no 4, pp. 18-21.

(2014), "Indicadores de Acceso Abierto. Evaluando el Crecimiento y Uso de los Recursos de Acceso Abierto de Regiones en Desarrollo. El Caso de América Latina", en J. P. Alperin; D. Babini; R. Fischman (eds.), Indicadores de Acceso Abierto y Comunicaciones Académicas en América Latina. Ciudad Autónoma de Buenos Aires, CLACSO.

. (2015). The Public Impact of Latin America's Approach to Open Access. Tesis (Doctoral), Universidad de Stanford.

; BABINI, Dominique; FISCHMAN, Gustavo (eds.). (2014), Open Access Indicators and Scholarly Communications in Latin America. Buenos Aires, CLACSO.

ALPERIN, Juan Pablo; FISCHMAN, Gustavo. (2015). Hecho en Latinoamérica: Acceso Abierto, Revistas Académicas e Innovaciones. Ciudad Autónoma de Buenos Aires, CLACSO.

ALPERIN, Juan Pablo; FISCHMAN, Gustavo; WILLINSKY, John. (2008). "Open Access and Scholarly Publishing in Latin America: Ten Flavours and a Few Reflections". Liinc em Revista, vol. 4, no 2, pp. 172-185.

(2011). "Scholarly Communication Strategies in Latin America's Research-intensive Universities". IESALC-Educación Superior y Sociedad, vol. 16, no 2.

ALTBACH, Philip. (2002), "Centers and Peripheries in the Academic Profession: The Special Challenges of Developing Countries", en P. Altbach (ed.), The Decline of the Guru: The Academic Profession in Developing and Middle-Income Countries. Boston, Center for International Higher Education, Boston College.

ARUNACHALAM, S. (2002), "Citation the Global Research Village: A View from the Periphery". Report, University of Arizona. Disponible en: http://hdl.handle.net $/ 10625 / 6007$

BABINI, Dominique. (2014a), “Universidades y Acceso Abierto: Hora de Tomar Protagonismo". Foro Revista Iberoamericana de Ciencia, Tecnología y Sociedad.

(2014b), “El Riesgo de que el Acceso Abierto Sea Integrado Dentro del Sistema Tradicional de Publicación Comercial - Necesidad de un Sistema Global no Comercial de Comunicaciones Académicas y Científicas". Revista Eletrônica de Comunicação, Informação e Inovação em Saúde, vol. 8, no 4, pp. 433-437.

DADOS - Revista de Ciências Sociais, Rio de Janeiro, vol. 61, nº 1, 2018 
BEIGEL, Fernanda. (2010), Autonomía y Dependencia Académica. Universidad e Investigación Científica en un Circuito Periférico: Chile y Argentina (1950-1980). Buenos Aires, Biblos.

(2012), “David y Goliath. El Sistema Académico Mundial y las Perspectivas del Conocimiento Producido en la Periferia". Pensamiento Universitario, Año 15, no 15, pp. 15-34.

. (2014), “Publishing from the Periphery: Structural Heterogeneity and Segmented Circuits. The Evaluation of Scientific Publications for Tenure in Argentina's CONICET". Current Sociology, vol. 62, no 5, pp. 743-765.

. (2015), “Culturas [evaluativas] Alteradas”. Política Universitaria, no 2, pp.11-21.

. (2016), "El Nuevo Carácter de la Dependencia Intelectual". Cuestiones de Sociología: Revista de Estudios Sociales, no 14.

__ ;SALATINO, Maximiliano. (2015), “Circuitos Segmentados de Consagración Académica: Las Revistas de Ciencias Sociales y Humanas en Argentina". Información, Cultura y Sociedad, no 32, pp. 7-32.

BIANCHETTI, Lucídio. (2010), “O Processo de Bolonha e a Intensificação do Trabalho na Universidade: Entrevista com Josep M. Blanch”. Educação e Sociedade, vol. 31, no 110, pp. 263-285.

;MACHADO, Ana Maria. (2007), Reféns da Produtividade: Sobre Produção do Conhecimento, Saúde dos Pesquisadores e Intensificação do Trabalho na Pós-Graduação. Trabalho apresentado na 30a Reunião Anual da Anped, 7-10 de outubro, Caxambu, Minas Gerais.

BURKHARDT, Frederick. (1960), "Inter-American Scholarly Communication in the Humanities and Social". The Library Quarterly, Information, Community, Policy, vol. 30, no 4, pp. 266-273.

CARVALHO NETO, Silvio; WILLINSKY, John; ALPERIN, Juan Pablo. (2016), “Measuring, Rating, Supporting, and Strengthening Open Access Scholarly Publishing in Brazil". Education Policy Analysis Archives, vol. 24, no 54.

CETTO, Ana María; ALONSO-GAMBOA, Octavio. (1998), “Scientific Periodicals in Latin America and the Caribbean: A Perspective". Interciencia, vol. 23, № 2, pp. 84-93.

CETTO, Ana María; HILlERUd, Kai-Inge. (1995), Publicaciones Científicas en América Latina. México, Fondo de Cultura Económica.

CETTO, Ana María; VESSURI, Hebe. (2005), “Latin America and the Spanish-speaking Caribbean", en M. El Tayeb; S. Schneegans (eds.), UNESCO Science Report. Paris, UNESCO.

CHATELIN, Yvon; ARVANITIS, Rigas. (1989). "Between Centers and Peripheries: The Rise of a New Scientific Community". Scientometrics, vol. 17, nos 5-6, pp. 437-452.

CHAUÍ, Marilena. (1989), “Produtividade e Humanidades”. Tempo Social, vol. 1, no2, pp. 45-71.

COIMBRA JR., Carlos. (2012), "Efeitos Colaterais do Produtivismo Acadêmico na Pós-Graduação". Cadernos de Saúde Pública, vol. 25, no 10, pp. 2092-2093. 
Más Allá de la Indexación: Circuitos de Publicación de Ciencias Sociales...

COLLYER, Fran. (2016), "Global Patterns in the Publishing of Academic Knowledge: Global North, Global South". Current Sociology, vol. 66, no 1, pp. 1-18.

CRANE, Diana. (1967), “The Gatekeepers of Science: Some Factors Affecting the Selection of Articles for Scientific Journals". The American Sociologist, vol. 2, no 4, pp 195-201.

GINGRAS, Yves. (2002), “Les Formes Spécifiques de L'internationalité du Champ Scientifique". Actes de la Recherche en Sciences Sociales, nos 141-142, pp. 31-45.

GODOY URZÚA, Hermán. (1969), "Social Science Communication in Latin America". Latin American Research Review, vol. 4, no 2, pp. 211-213.

GUÉDON, Jean-Claude. (2001), In Oldenburg's Long Shadow: Librarians, Research Scientists, Publishers, and the Control of Scientific Publishing. Washington, Association of Research Libraries.

(2008), "Open Access and the Divide between "Mainstream" and "Peripheral" Science", en M. Sueli; S. P. Ferreira; M. das Graças Targino (eds.), Como Gerir e Qualificar Revistas Científicas. Disponível em http:/ / eprints.rclis.org/10778/.

. (2011), “El Acceso Abierto y la División entre Ciencia Principal y Periférica”. Crítica y Emancipación, no 6, pp.135-180.

GUIMARÃES, J.A.; HUMANN, M. C. (1995), “Training of human-resources in science and technology in Brazil. The importance of a vigorous postgraduate program and its impact on the development of the country". Scientometrics, vol. 34, no 1, pp. 101-119.

HEILBRON, Johan. (2002), "La Bibliométrie, Genèse et Usages". Actes de la Recherche en Sciences Sociales, nos 141-142, pp. 78-79.

HOOD, William; WILSON, Concepción. (2001), "The Literature of Bibliometrics, Scientometrics, and Informetrics". Scientometrics, vol. 52, no 2, pp. 291-314.

LARIVIÈRE, Vincent; HAUSTEIN, Stefanie; MONGEON, Philippe. (2015), “The Oligopoly of Academic Publishers in the Digital Era". PLoS ONE, vol. 10, № 6.

MACHADO, Ana Maria Netto; BIANCHETTI, Lucídio. (2011), “(Des)fetichização do Produtivismo Acadêmico: Desafios para o Trabalhador-Pesquisador". Revista de Administração de Empresas, vol. 51, no 3, pp. 244-254.

MINGERS, John; LEYDESDORFF, Loet. (2015), “A Review of Theory and Practice in Scientometrics". European Journal of Operational Research, vol. 246, № 1, pp. 1-19.

MIRANDA, Elaine C. Pinto; MUGNAINI, Rogério. (2013), Scientific Policy in Brazil: Exploratory Analysis of Assessment Criteria. 14th International Society of Scientometrics and Informetrics Conference, 15-19 de julho, Viena.

MUGNAINI, Rogério. (2006), Caminhos para Adequação da Avaliação da Produção Científica Brasileira: Impacto Nacional versus Internacional, Tesis (Doutorado en Ciência da Informação), Universidade de São Paulo, São Paulo.

; DIGIAMPETRI, Luciano A.; MENA-CHALCO, Jesús P. (2014), “Comunicação Científica no Brasil (1998-2012): Indexação, Crescimento, Fluxo e Dispersão". Transinformação, vol. 26, no 3, pp. 239-252.

DADOS - Revista de Ciências Sociais, Rio de Janeiro, vol. 61, nº 1, 2018 
NASCIMIENTO, Carla; MUGNAINI, Rogério. (2016), Qualidade de Revistas Científicas: Um Estudo da Literatura Publicada entre 2003 e 2013. Trabalho apresentado no XVII Encontro Nacional de Pesquisa em Ciência da Informação, 20-25 de novembro, Universidade Federal da Bahia.

OLIVEIRA AMORIN, Keyla Mafalda de; DEGANI-CARNEIRO, Filipe; SILVA ÁVILA, Nathalia da. (2015), "Sistemas de Evaluación de las Revistas Científicas en Latinoamérica", en J. P. Alperín; G. Fischman (eds.), Hecho en Latinoamérica: Acceso Abierto, Revistas Académicas e Innovaciones Regionales. Ciudad Autónoma de Buenos Aires, CLACSO.

ORTIZ, Renato. (2009), La Supremacía del Inglés en las Ciencias Sociales. Buenos Aires, Siglo XXI.

REGO, Teresa Cristina. (2014), "Productivism, Research and Scholarly Communication: Between Poison and Medicine". Educação e Pesquisa, vol. 40, no 2, pp. 325-345.

SAFONOVA, María; SOKOLOV, Mikhail. (2013), "The Construction of the Academic World-System: Regression and Social Network Approaches to Analysis of International Academic Ties", en J. Gorraiz; E. Schiebel; C. Gumpenberger (eds.), Proceedings of the 14th International Society of Scientometrics and Informetrics Conference. Viena, Austrian Institute of Technology $\mathrm{GmbH}$.

SCHOTT, Thomas. (1991), “The World Scientific Community: Globality and Globalization". Minerva, no 29, pp. 440-462.

SGUISSARDI, Valdemar; SILVA JÚNIOR, João dos Reis. (2009), Trabalho Intensificado nas Federais: Pós-Graduação e Produtivismo Acadêmico. São Paulo, Xamã.

TORRES, Daniel; CABEZAS, Álvaro; JIMÉNEZ, Evaristo. (2013), “Altmetrics: Nuevos Indicadores para la Comunicación Científica en la Web 2.0". Comunicar, 41, pp. 53-60.

VELHO, Léa. (1986), "The Meaning of Citation in the Context of a Scientifically Peripheral Country". Scientometrics, vol. 9, no 1, pp. 71-89.

. (1992), “Indicadores de CYT e o seu Uso em Política Científica”. Sociedade e Estado, vol. 7, no 2, pp. 63-77.

. (1994), “Indicadores Científicos: Aspectos Teóricos y Metodológicos”, en E. Martínez (comp.), Interrelaciones entre la Ciencia, la Tecnología y el Desarrollo: Teorías y Metodologías. Montevideo, Unesco.

VESSURI, Hebe. (1987), “La Revista Científica Periférica. El Caso de Acta Científica Venezolana". Interciencia, vol. 12, no 3, pp. 124-134.

(1988), “Ciencia y Tecnología Hoy”. Interciencia, vol.13, no 6, pp. 317-318.

(1989), “Una Estrategia de Publicación Científica para la Fisiología Latinoamericana". Interciencia, vol. 11, no 1, pp. 9-13.

(1995), “Recent Strategies for Adding Value to Scientific Journals in Latin America". Scientometrics, vol. 34, no 1, pp. 139-161.

. (1996), "Scientific Cooperation among Unequal Partners: The Strait-Jacket of the Human Resource Base", en J. Gaillard (ed.), Coopérations Scientifiques Internationales. Les Sciences Hors D'occident au Xxe Siècle. Paris, ORSTOM. 
Más Allá de la Indexación: Circuitos de Publicación de Ciencias Sociales...

. (1997), "Sobre los Indicadores de Desempeño en las Ciencias Sociales", en H. Jaramillo; M. Albornoz (eds.), El Universo de la Medición: La Perspectiva de la Ciencia y la Tecnología. Bogotá, COLCIENCIAS/CYTED / TM Editores.

(2001), "Los Científicos Sociales en Venezuela. Perfil Bibliográfico e Implicaciones de Política”. Cuadernos del CENDES, año 18, no 48, pp. 89-121.

. (2007), O Inventamos o Erramos. La Ciencia como Idea-Fuerza en América Latina. Buenos Aires, Universidad Nacional de Quilmes.

. (2009), "The Current Internationalization of the Social Sciences in Latin America: Old Wine in New Barrels", en M. Kuhn; D. Weidemann (eds.), Internationalization of the Social Sciences and Humanities. Bielefeld, Transcript.

et al. (1984), La Ciencia Periférica. Ciencia y Sociedad en Venezuela. Caracas, Monte Avila Editores.

VESSURI, Hebe; GUÉDON, Jean-Claude; CETTO, Ana María. (2014), “Excellence or Quality? Impact of the Current Competition Regime on Science and Scientific Publishing in Latin America and its Implications for Development". Current Sociology, vol. 62, no 5, pp. 647-665.

VILAÇA, Murilo Mariano; PALMA, Alexandre. (2013), “Diálogo sobre Cientometria, Mal-Estar na Academia e a Polêmica do Produtivismo". Revista Brasileira de Educação, vol. 18, no 53 .

VOUTSSAS, Juan; CETTO, Ana María. (1996), “Electronic Publishing: Will it Reach the Whole World?", en D. Shaw; H. Moore (eds.), Electronic Publishing in Science, Proceedings of the Joint. Paris: ICSU Press/UNESCO Conference.

WOUTERS, Paul. (1999), "The Creation of the Science Citation Index", en M. E. Bowden et al. (eds.), Proceedings of the 1998 Conference on the History and Heritage of Science Information Systems. Medford, Information Today. 
RESUMO

Para além da Indexação: Circuitos de Publicação das Ciências Sociais na Argentina e no Brasil

Este artigo analisa 4.459 revistas de Ciências Sociais e Humanas da Argentina e do Brasil, no intuito de detectar segmentações na circulação associada às respectivas filiação disciplinar, acessibilidade e visibilidade, assim como ao seu enquadramento político-institucional. O conceito de circuitos de publicação é proposto como ferramenta analítica para se avançar além da lógica da indexação e complexificar as referências que associam a "excelência" a uma revista científica, a partir da sua inclusão em determinados bancos de dados e repositórios. A informação processada indica forte segmentação entre os circuitos locais, com uma maioria de publicações impressas de visibilidade reduzida e publicados por universidades tradicionais, um pequeno número inserido em circuitos da corrente principal de acesso restrito, além de, finalmente, outro grande grupo caracterizado pelo acesso aberto em circulação regional.

Palavras-chave: revistas científicas; indexação; circuito de publicação; Argentina; Brasil

\section{ABSTRACT}

Beyond Indexing: Social Sciences Publication Circuits in Argentina and Brazil

The following article analyzes 4,459 social sciences and humanities journals in Argentina and Brazil in order to detect segmentation in circulation associated with their disciplinary affiliation, accessibility-visibility and their political-institutional framework. The concept of publication circuits is proposed as a tool of analysis for advancing beyond the logic of indexing and to problematize references associating "excellence" with a particular scientific journal based on its inclusion in certain databases and repositories. The information processed indicates strong segmentation among local circuits, with most journals of scant visibility and published by traditional universities in paper format, a small number of limited access inserted in mainstream circuits, and finally, another large group characterized by easy access and regional circulation.

Key words: scientific journals; indexing; publication circuit; Argentina; Brazil 
Más Allá de la Indexación: Circuitos de Publicación de Ciencias Sociales...

\section{RÉSUMÉ}

Au-delà de l'Indexation: Circuits de Publication des Sciences Sociales en Argentine et au Brésil

Cet article analyse 4459 revues de sciences sociales et humaines de l'Argentine et du Brésil en vue de détecter des segmentations de la circulation associées à leur affiliation disciplinaire, leur accessibilité-visibilité et leur cadre politico-institutionnelle. Le concept de circuits de publication est proposé en tant qu'outil analytique pour dépasser la logique de l'indexation et mettre en perspective les références associant "l'excellence" d'une revue spécifique à son inclusion dans des bases de données déterminées. Les informations recueillies nous ont indiqué une forte segmentation entre les circuits locaux (avec une majorité d'éditions papier à faible visibilité publiées par les universités traditionnelles), un petit nombre de revues à l'accès restreint insérées dans les circuits principaux, et finalement, un autre grand groupe caractérisé par un accès ouvert et une circulation régionale.

Mots-clés: revues scientifiques; indexation; circuit de publication; Argentine; Brésil

\section{RESUMEN}

Más Allá de la Indexación: Circuitos de Publicación de Ciencias Sociales en Argentina y Brasil

Este artículo analiza 4459 revistas de ciencias sociales y humanidades de Argentina y Brasil con el fin de detectar segmentaciones en la circulación asociadas a su adscripción disciplinar, su acceso-visibilidad y su entramado político-institucional. El concepto de circuitos de publicación es propuesto como herramienta analítica para avanzar más allá de la lógica de la indexación y complejizar las referencias que asocian "excelencia" a una revista científica a partir de su inclusión en determinadas bases y repositorios. La información procesada indica una fuerte segmentación entre circuitos locales, con una mayoritaria cantidad de revistas editadas en papel, de escasa visibilidad y publicadas por universidades tradicionales, un pequeño número insertas en circuitos de la corriente principal y de acceso restringido y, finalmente, otro gran grupo caracterizado por el acceso abierto de circulación regional.

Palabras claves: revistas científicas; indexación; circuito de publicación; Argentina; Brasil

DADOS - Revista de Ciências Sociais, Rio de Janeiro, vol. 61, no 1, 2018 